\title{
Supplementary Information: Rigidity Dictates Spontaneous Helix Formation of Thermoresponsive Colloidal Chains in Poor Solvent
}

\author{
Bipul Biswas ${ }^{1,2}$, Debarshi Mitra ${ }^{3}$, Fayis $\mathrm{KP}^{1,2}$, Suresh Bhat ${ }^{1}$, Apratim Chatterji ${ }^{3,4, *}$, Guruswamy Kumaraswamy ${ }^{5, *}$ \\ ${ }^{1}$ PSE Division, CSIR-NCL Pune, Dr. Homi Bhaba Road, Pune-411008, India. \\ 2 Academy of Scientific and Innovative Research (AcSIR), Ghaziabad 201002, India \\ ${ }^{3}$ Dept. of Physics, IISER-Pune, Dr. Homi Bhaba Road, Pune-411008, India. \\ ${ }^{4}$ Center for Energy Science, IISER-Pune, Dr. Homi Bhaba Road, Pune-411008, India. \\ ${ }^{5}$ Chemical Engineering, Indian Institute of Technology Bombay, \\ Mumbai, 400076, India. * Address correspondence to these authors
}

(Dated: December 6, 2021)

\section{I.. LIST OF VIDEOS}

Video-1: Rigid colloidal polymer. Such chains show modest decrease in size but do not change their shape on heating. Thermal gradients set up during heating of the dispersion result in a small extent of convection of the chains. We select one chain with $\mu=0.95$ (as indicated in the video) and track it as the dispersion is heated.

Video-2: Semi-flexible colloidal polymer chains form helical structures on heating above LCST of PNIPAM. Thermal gradients set up during heating of the dispersion result in a small extent of convection of the chains. We select one chain with $\mu=0.85$ (as indicated in the video) and track it as the dispersion is heated.

Video-3: Flexible colloidal chains exhibit significant reduction in their mean size on heating at above the LCST of PNIPAM. Thermal gradients set up during heating of the dispersion result in a small extent of convection of the chains. We select one chain with $\mu=0.65$ (as indicated in the video) and track it as the dispersion is heated.

Video-4: Colloidal chain held isothermal at $45^{\circ} \mathrm{C}$. The helical structure is dynamical: helical segments form and unwind dynamically as a function of time. We track the time evolution of structure for one chain with $\mu=0.83$.

Video-5: Simulation video showing helix formation for chains of intermediate rigidity.

Video-6: A chain with right handed helicity is reconstructed as a $3 \mathrm{D}$ plot in a coordinate system comprising the spatial coordinates (XY) and image intensity (plotted along the $\mathrm{Z}$ axis). We plot data for points selected along the chain backbone, as explained in the main manuscript. As the chain is rotated, the helical conformation is viewed from different angles. We first view the chain normal to the helical axis, where the conformation appears sinusoidal. On rotating, the helical pitch can be observed. Finally, we view the conformation down the helical axis, where the projected structure appears circular.

Video-7: Rotation of chain showing left handed helical conformation, with views similar to those in Video-6.

\section{II.. MATERIALS AND METHODS}

\section{A. MATERIALS USED}

Polyethylene-glycol di-epoxide (PEG-di-epoxide, supplier specified molecular weight, $M_{n}=500 \mathrm{~g} / \mathrm{mol}$ ), allylamine, sodium dodecyl sulfate (SDS), Fluorescein isothiocyanate isomer and dialysis bags were obtained from Sigma-Aldrich and were used as received. $N$-isopropylacrylamide from Acros Organic, $N, N^{\prime}$ methylenebisacrylamide (BIS) and potassium peroxydissulphate (KPS) from Merck were used as received. Monodisperse polystyrene latex dispersions $(10 \% \mathrm{w} / \mathrm{v})$ with a manufacturer specified size of $1.08 \mu \mathrm{m}$ were obtained from Microparticles GmbH. ITO coated cover slips from Helix Biotech Instruments and indium tin oxide (ITO) coated glass slides from Technistro were used as received.

\section{B. SYNTHESIS OF PNIPAM MICROGELS}

Poly( $N$-isopropylacrylamide co-allylamine) microgels were prepared via a free radical precipitation polymerization. The polymerization reaction was carried out in a $250 \mathrm{~mL}$ three-neck round bottom (RB) flask equipped with a mechanical stirrer, a condenser and an argon inlet. The RB flask was kept in an oil bath throughout the reaction to maintain the reaction temperature at $60^{\circ} \mathrm{C}$. A bottle of de-ionised water purged with argon gas for $1 h$, was used for preparing solutions. In a typical experiment, the purified reactants, monomer $\mathrm{N}$-isopropylacrylamide $(1.935 g)$, a cross-linker $N, N^{\prime}$ - methylene bisacrylamide (BIS) $(0.215 \mathrm{~g}, 10 \%$ of the total monomer) and surfactant sodium dodecyl sulfate (SDS) $(0.03 g)$ were mixed in $115 \mathrm{~mL}$ of argon purged deionized water at $40^{\circ} \mathrm{C}$ by stirring at $100 \mathrm{rpm}$ for 30 minutes under inert atmosphere. After complete mixing of the reactants, the temperature was increased to $60^{\circ} \mathrm{C}$ and co monomer allylamine $(0.107 \mathrm{~g} 5 \%$ of the total monomer) was added to the mixture. After waiting for thermal equilibration, the initiator KPS $(0.025 \mathrm{~g}$ in $10 \mathrm{~mL}$ of water), was added to the reaction mixture. The reaction mixture changed from transparent to turbid after addition of initiator. The reaction was allowed to proceed for 6 hours to ensure 
complete conversion. After 6 hours, the temperature was reduced to $25^{\circ} \mathrm{C}$ and the product (micro-gel) was stirred overnight at $100 \mathrm{rpm}$. The micro-gel dispersion was purified by dialysis against deionized water for about two weeks to separate unreacted reactants and surfactant from the micro-gel dispersion. We also tagged FITC on PNIPAM microgel according to the reported article[1]. In short, $10 \mu L$ of $0.04 \mathrm{mg} / \mathrm{mL}$ FITC solution was added in $1 m L$ of synthesized PNIPAM dispersion.

\section{SAMPLE PREPARATION}

We dilute $5 \mu L$ of PNIPAM micro-gel dispersion $\left(\phi_{\text {eff }}=0.153\right)$ in $200 \mu L$ of DI water and sonicate for 2 minutes. We dilute $1 \mu L$ of PS particle dispersion (containing $\sim 4.8 \times 10^{7}$ PS latex particles) in $200 \mu L$ of DI water. Then, we add the dilute PS dispersion to the dilute PNIPAM micro-gel dispersion and sonicate for 5 minutes. The positively charged PNIAPM microgel particles adsorb on the negatively charged surface of the PS particles. This dispersion was centrifuged at $4000 \mathrm{rpm}$ for 7 minutes and the supernatant was removed to separate unabsorbed PNIPAM microgel particles. Then, we add $150 \mu L$ of DI water/glycerol mixture (volume ratio of water and glycerol is $5: 1$ ) to further dilute the dispersion and sonicate for 5 minutes. The density of the fluid is adjusted so that it is close to that of the PS latex, viz. $\sim 1.05 \mathrm{~g} / \mathrm{cm}^{3}$. We prepare a chamber using parafilm on the ITO glass slide (length $\sim 4 \mathrm{~mm}$, width $\sim 4 \mathrm{~mm}$ and height $\sim 100 \mu m)$. We take $20 \mu L$ dispersion containing PNIPAM microgel adsorbed PS particles and add $0.5 \mu \mathrm{L}$ of cross-linker followed by sonication for 2 minutes. This was transferred into the chamber and covered with another ITO coated glass slide on top. We ensure that there is no air bubble trapped in the chamber. We seal this chamber properly so that there is no leakage during the experiment.

\section{PREPARATION OF LINEAR CHAIN BY AC FIELD}

In all experiments, the electric field is imposed using an amplifier (PZD 350 from Trek, USA) and a function generator (AFG1022 from Tektronix) and is monitored using a digital oscilloscope (TSB1102B from Tektronix). We apply AC field with $E_{r m s}=0.25 \mathrm{~V} / \mu \mathrm{m}, f=250 \mathrm{kHz}$, where $E_{r m s}$ is the root mean square electric field across the ITO coated glass slides sandwiching the dispersion and $f$ is the frequency. We observe the dispersion using an optical microscope, and observe that all the particles assemble into linear chains along the field direction within 2-3 minutes of imposing the field. [2, 3] We keep the electric field on for $12-25$ minutes to allow crosslinking through the amine groups on the PNIPAM microgel particles and then turn off the field. We observe that this results in the formation of permanent chains dis- persed in the solvent. Since the density of the solvent is closely matched with the PS particles, the chains do not settle over long periods of time ( $2 \mathrm{hrs})$. We change the crosslinking time to vary the rigidity of the chains. To make the rigid chains we allow crosslinking to proceed for $20-25$ minutes. Under these conditions, we observed that $\sim 70 \%$ chains formed are rigid and $\sim 30 \%$ are semiflexible. To prepare flexible chains, we crosslink for $12-15$ minutes. Under these conditions, $\sim 60 \%$ chains are relatively flexible and some semiflexible and a few rigid chains also form. Semiflexible chains are prepared by crosslinking for $15-20$ minutes. Under these conditions, $\sim 60 \%$ chains formed are semiflexible, $\sim 20 \%$ flexible and $\sim 20 \%$ rigid. Schematic of PNIPAM coated particles with cross-linker and the formation of linear chains of particles on application of an AC electric field are depicted schematically in FIG. 1 in the main manuscript.

\section{E. IMAGING}

We use a confocal laser scanning microscope (CLSM, Carl Zeiss model-LSM710). For experiments to calculate the temperature dependent pair correlation function of the thermoresponsive (PNIPAM coated PS) "monomeric beads", we use the confocal mode of the microscope. To estimate the bonding strength between two monomers in a chain of bonded beads, and to estimate the bending rigidity between two bond vectors, we use the optical mode of microscope with a $63 \mathrm{X}$ objective. For all other experiments, we use a $20 X$ objective.

\section{F. COATING OF PNIPAM ON PS COLLOIDS}

FITC tagged PNIAPM microgel are coated on the PS colloids (rhodamine tagged) and used to prepare colloidal chains. The chains were imaged using confocal microscopy with two different excitation wavelengths $(\lambda=543 \mathrm{~nm}$ and $\lambda=490 \mathrm{~nm})$. In FIG. S1 the left panel image is obtained by exciting at wavelength $490 \mathrm{~nm}$ where only the shell layer of FITC-tagged PNIPAM is visible. In FIG. S1 the right panel image is obtained by exciting at wavelength $543 \mathrm{~nm}$ where Rhodamine dyed PS colloids are visible. From FIG. S1 we observe that the PNIPAM microgels appear to be uniformly coated on the PS colloids.

\section{G. CHARACTERIZATION OF MICROGEL PARTICLES}

The microgel particles were characterized by dynamic light scattering (DLS) at very dilute suspension concentrations (volume fraction $\sim 10^{-3}$ ) in order to determine the temperature dependence of their size. We assume that the micro gels are sufficiently dilute that 

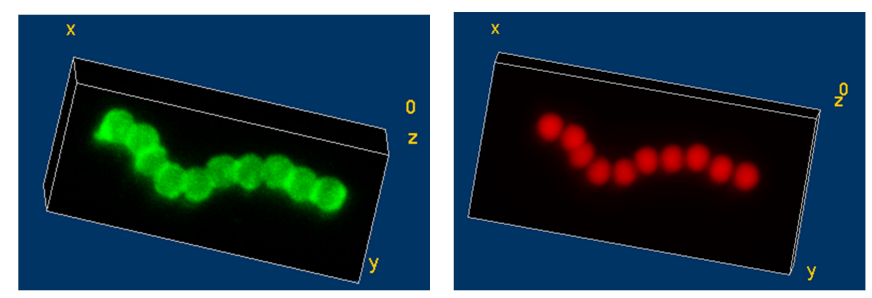

FIG. S1. Confocal image of the PNIPAM coated colloidal chains. (Left panel) excitation wavelength is $490 \mathrm{~nm}$ and we observe the PNIPAM microgels and (right panel) excitation wavelength is $543 \mathrm{~nm}$ and only Rhodamine B dyed PS colloids are visible. Note that each colloidal particle has a (manufacturer specified) size of $1.08 \mu \mathrm{m}$.

there are no two-body interactions at this concentration (including electrostatic interactions). The zeta potential of the micro-gel particles is $+15 \mathrm{mV}$. We performed DLS measurements at a fixed scattering angle, $2 \theta=90^{\circ}$, where $2 \theta$ is the scattering angle and is related to the scattering vector as $q=4 \pi n \sin \theta / \lambda$, where $\lambda(=633 \mathrm{~nm})$ is the wavelength of laser light in vacuum and $n$ is the refractive index of the solvent. We analysed the intensity-intensity autocorrelation function using a second-order cumulant method[4] and obtained the hydrodynamic diameter $\left(D_{h}\right)$ using the Stokes-Einstein equation $\left(D_{o}=k_{B} T / 3 \pi \eta D_{h}\right.$, where $D_{o}$ is the diffusivity from DLS, $k_{B}$ is the Boltzmann constant, $T$ is the temperature of the solvent and $\eta$ is the viscosity of the solvent). The hydrodynamic diameter $\left(D_{h}\right)$ of the microgel particles decreases with increasing temperature (FIG. S2) confirming the thermo-responsive nature of the PNIPAM micro-gels. We define the transition temperature as the mid-point of the onset temperature for decrease in $D_{h}\left(=25^{\circ} \mathrm{C}\right)$ and the temperature where $D_{h}$ saturates $\left(=39^{\circ} \mathrm{C}\right)$. Based on this definition, the LCST transition occurs at $32^{\circ} \mathrm{C}$.
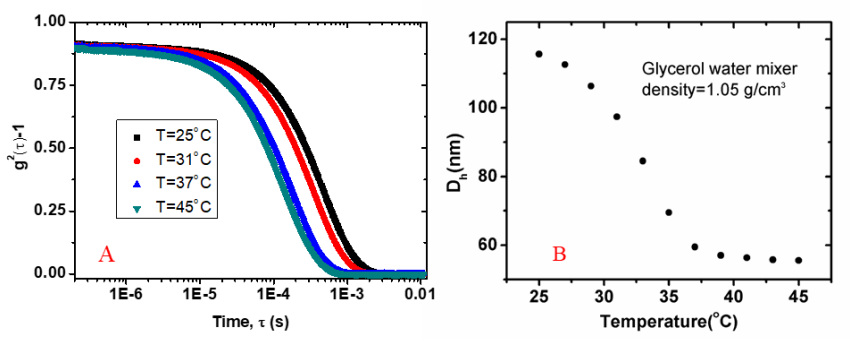

FIG. S2. (A) $g^{2}(\tau)-1$ vs t plot of DLS data. (B)Temperature dependence of the average hydrodynamic diameter $\left(D_{h}\right)$. On heating, the microgel particles exhibit a transition from a swollen state to dehydrated state. Each point represents the equilibrium size of the micro-gel at that temperature. We wait for at least 25 minutes at each temperature for the sample to equilibrate before measuring $D_{h}$.

\section{H. AGGREGATION OF THERMORESPONSIVE COLLOIDS}

To characterize the temperature dependent behaviour of the PNIPAM coated PS latex, we visualize the aggregation of colloidal particles, using CLSM. Suspensions were kept between a glass slide and a cover slip separated by a spacer of $120 \mu m$ thickness. We performed our experiment in bulk suspensions, by focusing at a plane at least $10 \mu \mathrm{m}$ below the cover slip. We collected a series of images in the $x-y$ plane at different temperatures, after allowing sufficient time for equilibration of the sample (FIG. S3). We perform experiments in 2D since confocal scanning in the $\mathrm{z}$ direction takes a long time during which the colloids diffuse. Thus, confocal scanning microscopy is too slow to allow us to track the motion of $1 \mu \mathrm{m}$ Brownian colloids in 3D. We present confocal micrographs of PNIPAM coated particles as a function of temperature in FIG. S3.
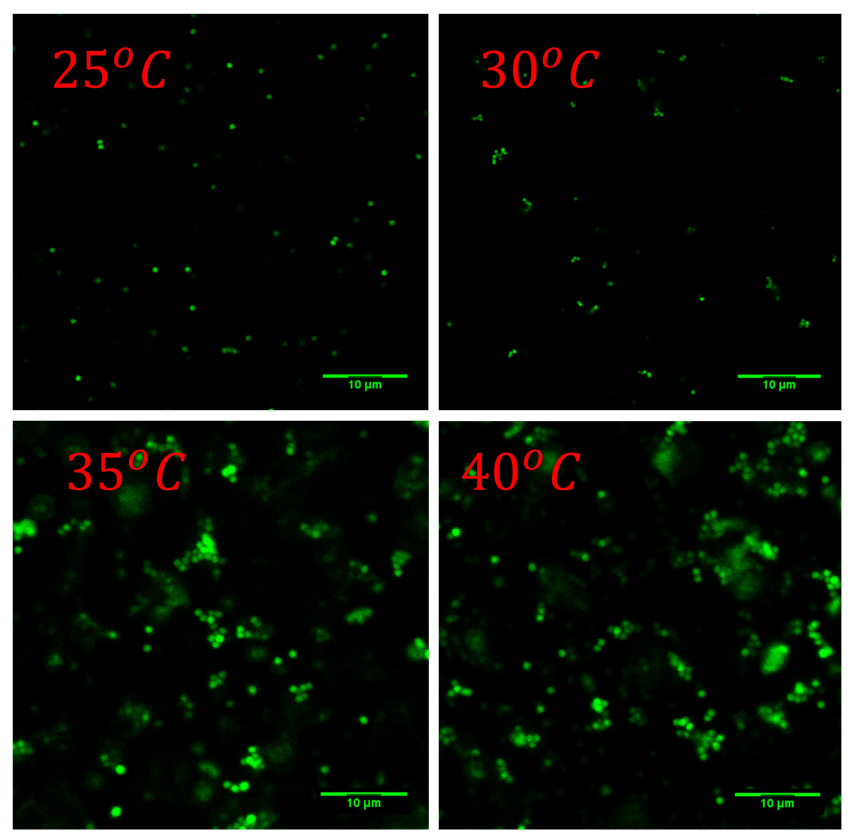

FIG. S3. (Confocal micrographs of PNIPAM coated particles as a function of temperature. At temperatures below the LCST (for example, at $25^{\circ} \mathrm{C}$ ) particles are well dispersed. As the temperature increases above the LCST, PNIPAM microgels dehydrate and the colloids start aggregating. With increase in aggregate size, the Brownian motion gets sluggish - thus, this process results in the formation of multiple small clusters instead of a giant cluster.

\section{FINDING END TO END DISTANCES AND CONTOUR LENGTH}

Images were uploaded in ImageJ and we fit the colloidal chain using the spline fit option to determine the contour length of the chains and estimate the coordinate 
of the end points of the chains (shown in FIG. S4)[5]. We measured the contour length and end to end distances using at least 10 indeppendent images and found the standard deviation is less than $1 \%$.
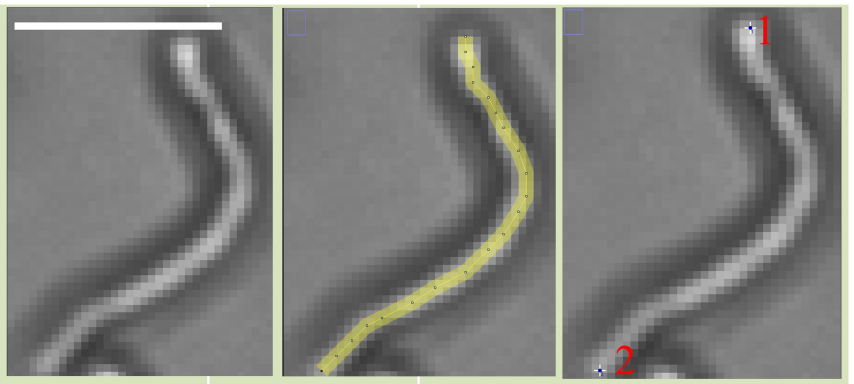

FIG. S4. Extracting the end to end distances and contour length of the colloidal chains. The scale bar is $10 \mu \mathrm{m}$. The middle image shows a spline-fit to the backbone, used to calculate the contour length and the image on the right shows the end points, used to determine the end-to-end length.

\section{J. CALCULATION OF RIGIDITY, SPRING CONSTANT AND BENDING RIGIDITY}

We control the rigidity of chain by varying the crosslinking time (see details in Section II.D). We measure rigidity $\mu$ based on the equation $\mu L^{2}=\left\langle R^{e}(t)^{2}\right\rangle$, where $R^{e}(t)$, is the end to end distance at time t, $\left\langle R^{e}(t)^{2}\right\rangle$ is the average of the square of the end to end distance of the chain and $L$ is the contour length of the chain. We also measure the end to end distance in $2 \mathrm{D}$, $R^{e}(t)=\sqrt{\left(x_{0}-x_{\text {end }}\right)^{2}+\left(y_{0}-y_{\text {end }}\right)^{2}}$, where $\left(x_{0}, y_{0}\right)$ and $\left(x_{\text {end }}, y_{\text {end }}\right)$ are the positions of the end points of the chain in an arbitrary reference frame at any time, $t$. To estimate the spring constant and bending rigidity of the thermo-responsive colloidal chains we performed experiments at $25^{\circ} \mathrm{C}$ with $63 \mathrm{X}$ objective (S5A. Individual colloidal particles can be clearly resolved in the high-resolution images. We use the imfindcircles function in MATLAB to find center of mass position of individual colloids with subpixel resolution (S5B). We now present the distribution of the center to center distance between bonded colloids for chains with different rigidity (FIG. S5C). We fit this distribution to a Gaussian, $P(r) \sim \exp ^{\left[-k\left(r-r_{c}\right)^{2} /\left(k_{B} T\right)\right]}$ and obtain the spring constant, $k$, that characterizes the bonding between the colloids. As the value of $\mu$ increases from 0.63 to 0.81 to 0.94 , we find that $k$ increases from $56.02 k_{B} T \mu m^{-2}$ to $76.34 k_{B} T \mu m^{-2}$ to $119.94 k_{B} T \mu m^{-2}$ respectively. Next, we obtain bond angle distributions (as indicated in the schematic FIG. S5D, inset) for chains with different rigidity, $\mu$. Again, we fit this distribution to a Gaussian form, $P(\theta) \sim \exp ^{\left(-\kappa \theta^{2} / k_{B} T\right)}$ and obtain the bending rigidity, $\kappa$. As $\mu$ increases from 0.49 to 0.81 to 0.94 there is an increase in bending rigidity $(\kappa)$ from $0.8 \times 10^{-3} k_{B} T(\theta)^{-2}$
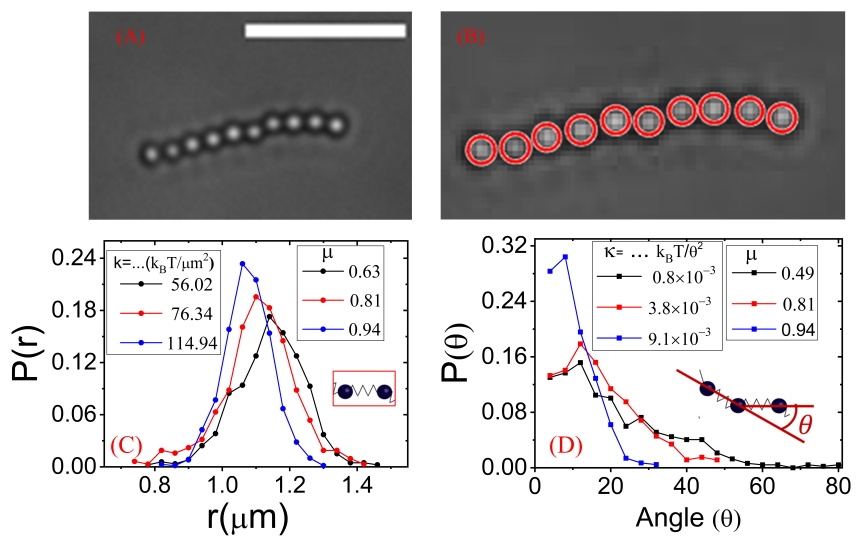

FIG. S5.

(A) Image captured with $63 \mathrm{X}$ objective and scale bar is $10 \mu \mathrm{m}$. (B) We use imfindcircle function in MATLAB to find centre of mass position of individual colloids. (C) Inter-particle distances are narrowly distributed for rigid chain whereas the distribution for relatively flexible chains is wider. (D) Similarly, the distribution of angles between two bond vectors about the mean value is narrow for rigid chains and wider for relatively flexible chains.

to $3.8 \times 10^{-3} k_{B} T(\theta)^{-2}$ to $9.1 \times 10^{-3} k_{B} T(\theta)^{-2}$.
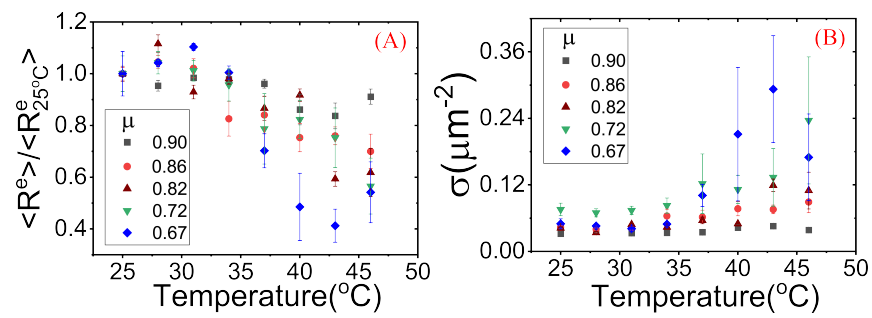

FIG. S6. (A) Variation of end to end distances of chain (normalized with the average end to end distance at $25^{\circ}$ ) with temperature. (B) Variation of density $\left(\sigma=N /\left\langle\left(R^{e}\right)^{2}\right\rangle\right)$ of particles with temperature for chains with different $\mu$. It is seen that there is a drastic increase in $\sigma$ for flexible chains at above $35^{\circ} \mathrm{C}$, whereas there is almost no change in monomer density $(\sigma)$ for the most rigid chains.
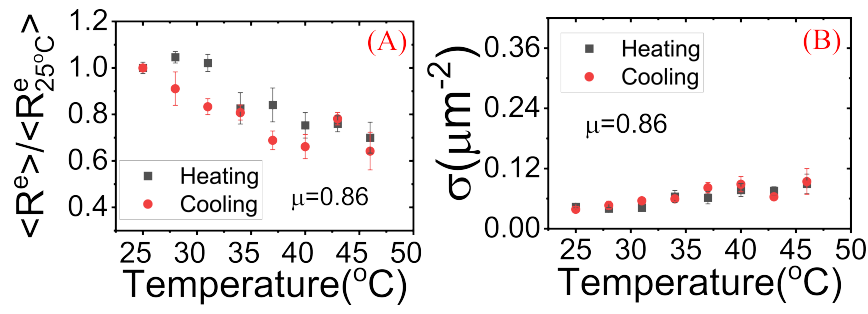

FIG. S7. (A) Variation of end to end distances of chain (normalized with the average end to end distance at $25^{\circ} \mathrm{C}$ ) with temperature during a heat-cool cycle. (B) Variation of density $\left(\sigma=N /\left\langle\left(R^{e}\right)^{2}\right\rangle\right)$ with temperature during a heatcool cycle. We observe no significant hysteresis during the heat-cool cycle. Samples are heated (or cooled) and are held isothermal for several minutes before measurements are made. 


\section{K. DEFINITION OF HELIX}

We use MATLAB to obtain the intensity along the contour length of an optical microscopy image of a colloidal chain. We define a helical segment as one where the image intensity varies periodically (low-high-low or highlow-high), along the contour length. We analyse only those images for chains that are nearly horizontal.Also, we use multi-points selection tool in ImageJ to extract the co-ordinates and corresponding intensity along the contour length of the chains and plot the intensity as a function of contour length (see inset in FIG. S8A-C). For the microscope lamp settings used in our experiments, and based on our experience of imaging multiple samples, we consider only intensity variations of larger than 30 (in the arbitrary units used in all our experiments) as significant. FIG. S8A represents an optical microscopy image of a rigid chain (Regime-III). From the inset, we note that there are no systematic intensity variations along the contour length. In the inset of FIG. S8B, we observe periodic intensity variations along the contour length and the amplitude of the variation exceeds 30 units. We classify this as a chain with a helical conformation. In FIG. S8C inset, we observe low amplitude intensity variations (amplitude $<30$ ) along the contour of a flexible chain (Regime-I). Further, we note that if any three successive low-high-low or high-low high intensity points fall on a straight line, then we consider that that segment does not represent a helix. To be assigned as a helix, the intensity should vary between extrema by $>30$ units and the spatial location of three consecutive extrema must not be collinear. In the image FIG. S8C and FIG. S9C, though there are some local minimum of intensity along the contour length, the intensity difference between high and low intensity points is less than 30 units and successive low-high-low or high-low-high points are approximately collinear. Therefore, we consider that this image does not correspond to a helical conformation.

\section{IDENTIFYING HELICAL CONFORMATIONS AND HANDEDNESS}

We use a $20 \mathrm{X}$ objective to capture video so that the entire chain can be visualized in one frame. Therefore, we cannot distinguish individual monomers. We analyze optical microscopy images of colloidal chains using ImageJ (FIG. S10A, C) and use the multi-point selection tool to locate the co-ordinates along the chain backbone and the corresponding intensity values. We identify successive high and low intensity points and manually select one or two points between these extrema. Typically, the distance $(\mathrm{d})$ between two successive intensity extrema is between $2 \mu m$ to $6 \mu m$. If $2 \mu m<d<4 \mu m$, we select one point between the extrema, while if $4 \mu m<d<6 \mu m$, we select two equispaced points between the extrema. We number each point sequentially and plot the image intensity as a function of the $\mathrm{x}, \mathrm{y}$ co-ordinates of points. We

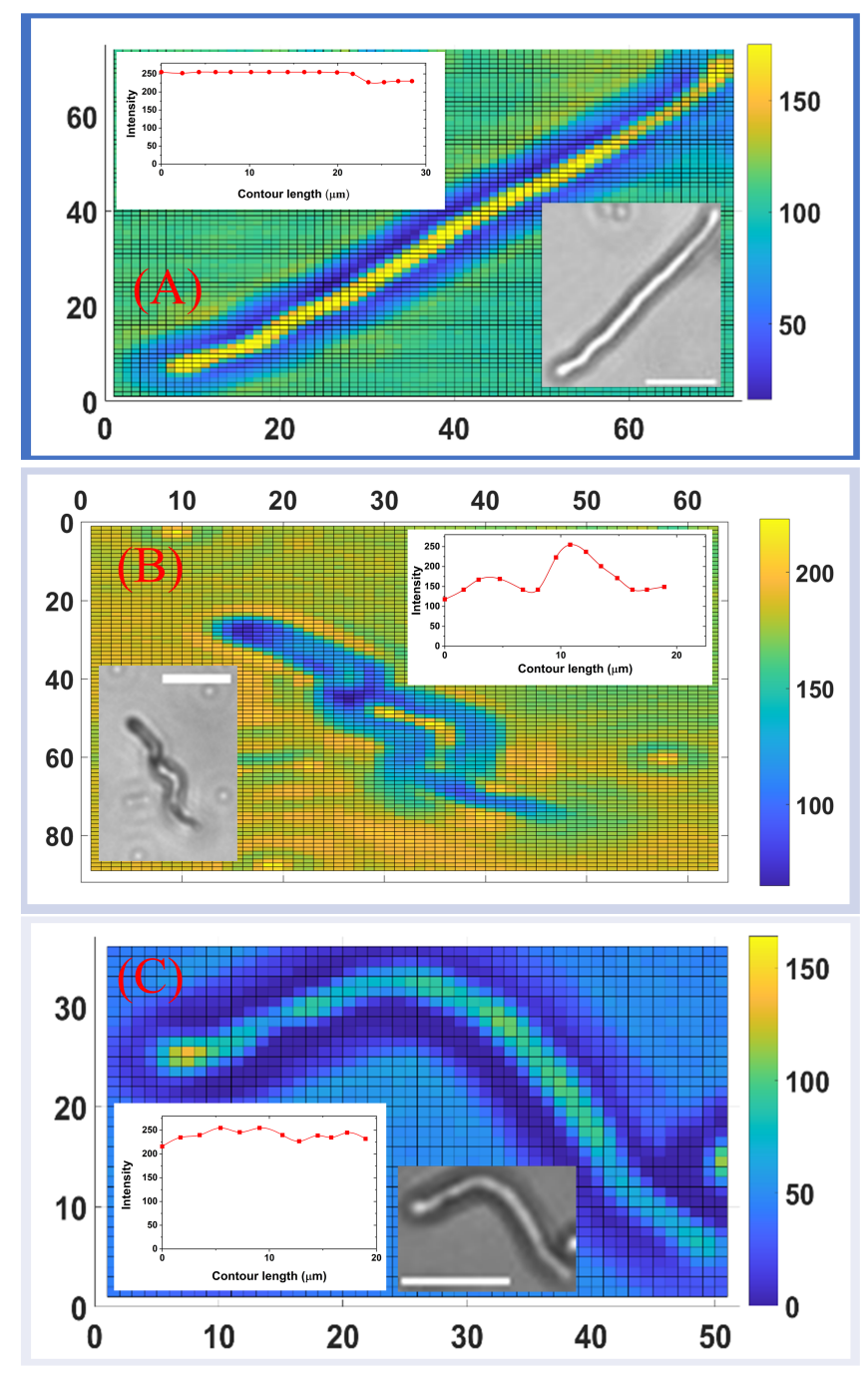

FIG. S8. Intensity variations of colloidal chains. (A) There is no intensity variation along the chain axis of a rigid chain.(B) We classify a chain as helical based on periodic intensity variations along the chain axis and when the spatial location of three consecutive extrema are not collinear. (C) Bent (but not helical) chain: Intensity variations along the chain are low amplitude ( $<30$ units). Scale bars in all figures are $10 \mu \mathrm{m}$. In all the images, the inset shows the intensity variations along the contour length of the corresponding chain.

measure the intensity values along the chains (marked using a red box in the image) where we see the periodic variations of intensity, along the direction marked by the arrow (FIG. S10A, C). The chains imaged are in the focal plane - therefore, as the helix winds out of the focal plane, there is a change in the measured intensity. Therefore, we use intensity as a proxy of the Z-coordinate. We observe that chains that exhibit periodic intensity variations in the 2D images are organized as helices in (XY-Intensity) $3 \mathrm{D}$ plots. We connect two consecutive points in the image through a "bond" vector, and observe that the bond vectors progress in helical fashion. This also allow us to 

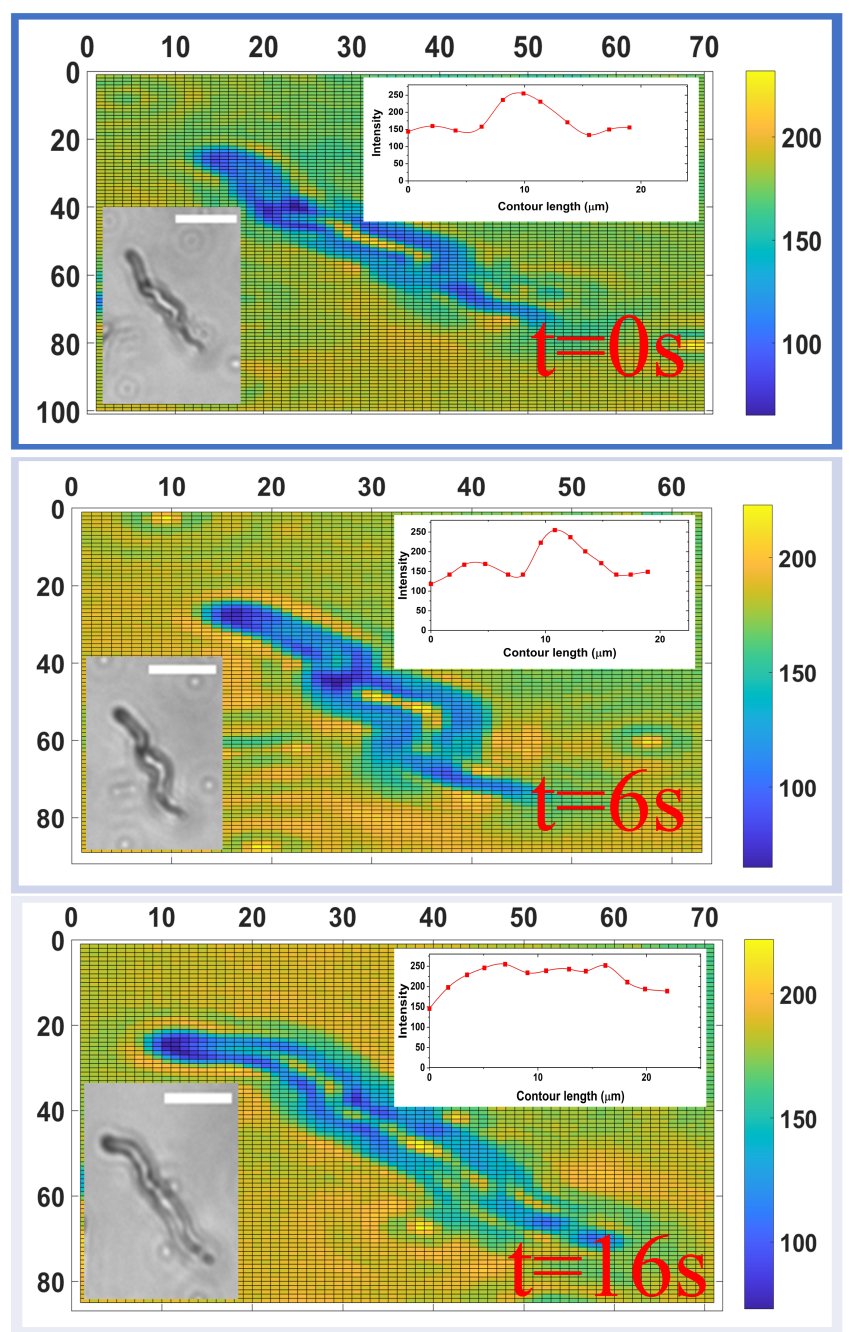

FIG. S9. Starting from an arbitrary time $t=0 \mathrm{~s}$, we present structural changes of a chain as time progresses. The helical structure appears $(t \sim 5 s$ and subsequently disappears $(t \sim 16 s)$, showing the dynamical nature of helical structure formation in the experiments. Intensity variations along the contour length of the corresponding chains are shown in inset. Scale bars in all figures are $10 \mu \mathrm{m}$.

infer the handedness of the helical structure. For example, (FIG. S10A, B) represents a right handed helix and (FIG. S10C, D) represents a left handed helix.

\section{MONOMER FRACTION IN HELICAL CONFORMATION AND PITCH LENGTH}

We estimate the contour length of the chain (shown in FIG. S4). Similarly, we estimate the length the chain segment in a helical conformation (red lines, shown in FIG.S11A, lower inset) from 2D images using the protocol described in the previous section. We divide the contour length and the helical length, respectively, by the size of an individual colloid to estimate the total

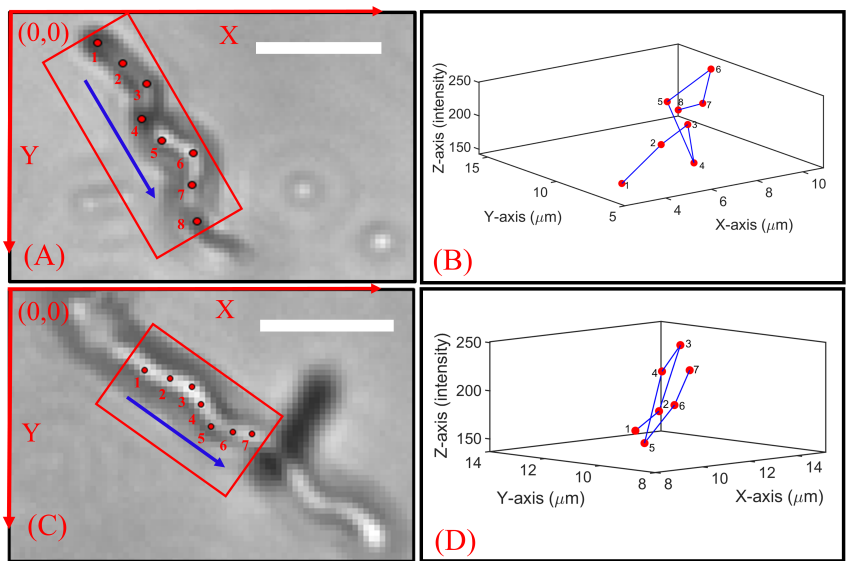

FIG. S10. (A) 2D optical microscopy images of a chain showing helical conformation. Image J is used to obtain $\mathrm{X}, \mathrm{Y}$ coordinates and image intensity of points on the backbone of the colloidal chains. (B) A 3D plot of image intensity (plotted along the $\mathrm{Z}$ axis, labelled I) as a function of the $\mathrm{X}, \mathrm{Y}$ coordinates of selected points along the chain shows the helical structure of the colloidal chains. Using intensity as a proxy for the $\mathrm{Z}$ coordinate shows that the chain adopts a right handed conformation. The colour bar represents the intensity scale. Similarly (C) and (D) represent an optical image and XY-I constructions of a chain that forms a left handed helix. The scale bars in $(\mathrm{A})$ and $(\mathrm{C})$ are $10 \mu \mathrm{m}$.

number of particles in a chain $(\mathrm{N})$ and the number of monomers in helical segments. To find the fraction of a chain that form helices we divide the helical segment length by the contour length. We obtain the pitch of the helix as the distance between two consecutive high intensity points or two consecutive low intensity points. The points in green in FIG.S11A lower inset, are low intensity points and distance between two successive points is measured as the pitch length.

The helical structure changes with time. We observe the helical structure as a function of time and present the helical pitch for 2 chains, with $\mu=0.88$ and 0.85 (FIG.S11B). We follow a chain as its helical structure unwinds (cycle 1) and again, after it reforms into a helix (cycle 2). We obtain the pitch for each helical turn along the chain and plot it as a point on FIG.S11B. Similarly, we plot the helical pitch for each turn of the other chain as a function of observation time. We observe that the pitch length varies over a range from $4 \mu m$ to $10 \mu m$, for the chains observed.

\section{III.. SIMULATION RESULTS}

\section{A. Mapping of $\epsilon_{b}$ to $\mu$}

In our simulations, the measure of the rigidity of the polymeric chain is controlled by the value of parameter $\epsilon_{b}$. However in the experiment, the rigidity of the chains 

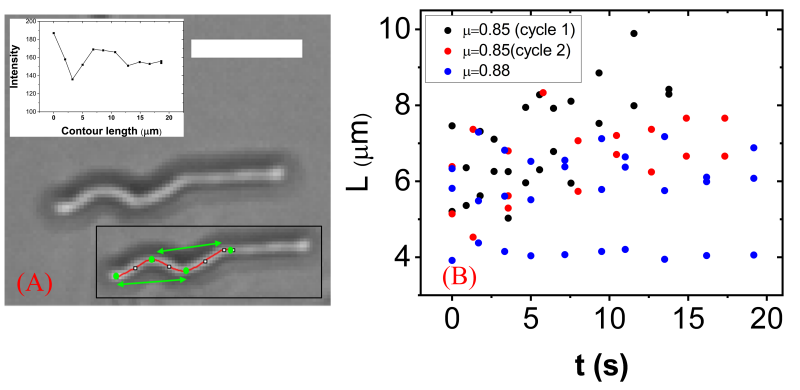

FIG. S11. (A) A segment of a chain (marked using a red line along the chain contour, lower inset) forms a helix (lower inset). The distance between two successive low or two high intensity points is represented as pitch length. In the image high and low intensity points are marked in green and the distance between two consecutive extrema are marked as the pitch lengths of the helical structure. In the inset at the top, the intensity variation along the contour length of the chain is plotted.(B) Pitch length distribution. Black and red points are from the same chain. Helical segments form and disappear, and reform as a function of time. The pitch lengths obtained from analyzing two different chains which form helices are shown in plot. Pitch length varies from $4 \mu \mathrm{m}$ to $10 \mu \mathrm{m}$. Scale bar is $10 \mu m$.

are characterized using the dimensionless parameter $\mu$ as defined previously.

To establish a correspondence between $\epsilon_{b}$ and $\mu$, we use 10 independent simulation runs for each value of $\epsilon_{b}$ in the absence of hydrophobic contractile forces. We take the time average value from each of these 10 independent runs to compute the mean square end-to-end length, which is then used to calculate the parameter $\mu$ in simulations, by averaging over different independent runs. Fig.S12 shows the computationally calculated $\mu$ values corresponding to $\epsilon_{b}$, which is a simulation parameter. Note that the values of $\mu$ calculated from our simulations have similar values as obtained from experiments. As expected the value of $\mu$ increases as polymeric chains becomes more rigid. However we do not have chains of $\mu \sim 0.7$ due to reasons outlined in the 'Simulation Details' section of the main manuscript.

\section{B. $H_{2}$ and $H 4$ for different chain lengths of the polymeric chain}

We quantify helicity using two order parameters $\mathrm{H} 2$ and $H 4$ as described in the main text. We show in Fig.S13 that we indeed obtain helices both for chain lengths of 40 and 25 monomers respectively when there is hydrophobic monomer-monomer attraction.

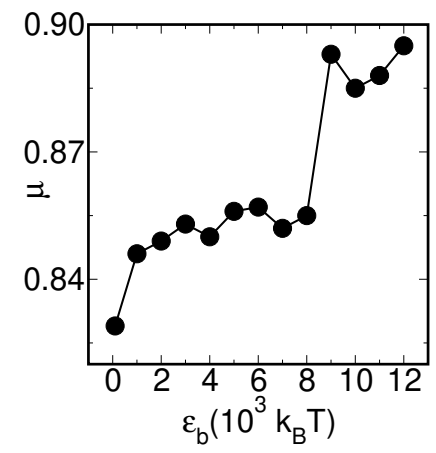

FIG. S12. To provide a mapping of experimental measurements to theoretical modeling studies we show the rigidity $\mu$ corresponding to different values of $\epsilon_{b}$.
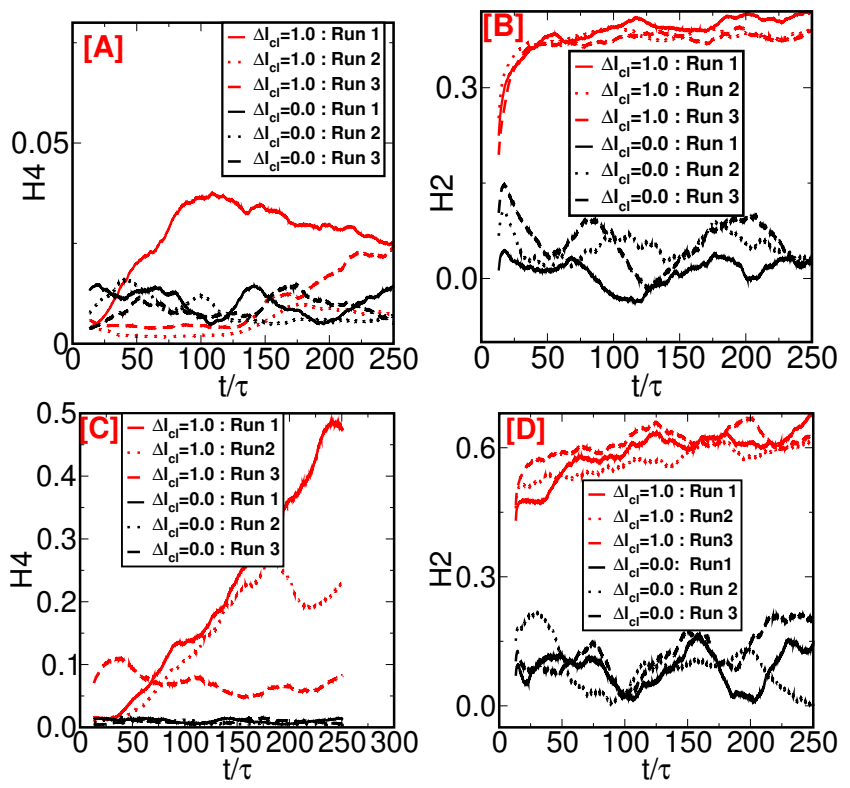

FIG. S13. (A) H4 running averaged over $25 \tau$ and (B) $H 2$ running averaged over $25 \tau$, as a function of time for $\kappa_{c l}=100 k_{B} T / a^{2}$ and $\epsilon_{b}=5000 k_{B} T(\mu \sim 0.8)$ with 3 independent runs for chains of 40 colloidal monomer, under hydrophobic contractile forces with $\Delta \ell_{c l}=1$ (measured in units of $a$ ). For comparison we also show 3 independent runs, corresponding to identical polymeric chains of colloids with identical parameters, but without hydrophobic attraction, i.e $\Delta \ell_{c l}=0$. Subfigures (C) and (D) show $H 2, H 4$ data for the same parameters as in (A) and (B) but for a shorter chain with only 25 monomers.

\section{Decrease in the end to end distance of the polymeric chain}

We investigate the decrease in the end to end distance of the polymeric chain as a consequence of hydrophobic contractile forces for two different values of $\epsilon_{b}$ (and the corresponding values of $\mu$ ). To that end, we show the end to end distance scaled by the contour length, $R_{e e} / R_{c}$ in Fig.S14. For each value of $\epsilon_{b}$ we obtain $R_{e e} / R_{c}$ by averaging over 10 independent realizations. Just as in 

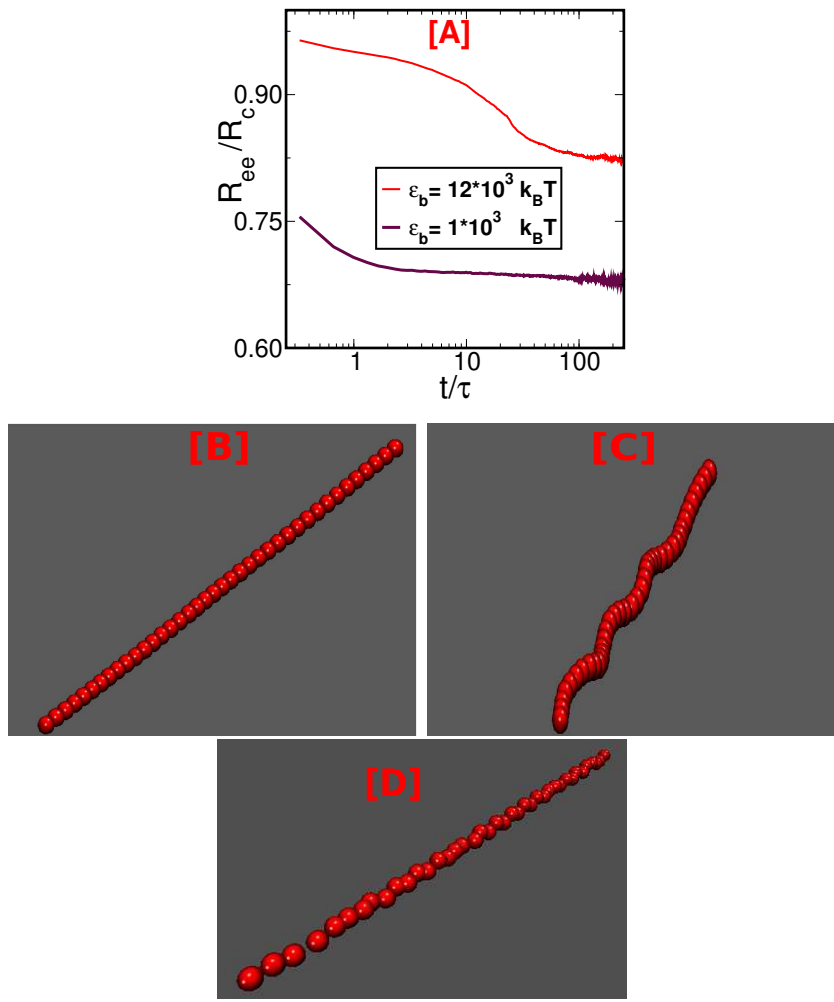

FIG. S14. (A) The end to end distance $R_{e e}$ (as a fraction of contour length $R_{c}$ ) is plotted as a function of time for chains of two different flexibilities of $\epsilon_{b}=10^{3} k_{B} T$ and $\epsilon_{b}=$ $16 \times 10^{3} k_{B} T$, respectively, when hydrophobic compression is introduced at time $t=0$. (B) Representative snapshots of the conformations of the polymeric chain for the stiff chain: $\epsilon_{b}=12 \times 10^{3} k_{B} T$, the (C) semi-flexible chain with $\epsilon_{b}=$ $5 \times 10^{3} k_{B} T$, and (D) the relatively flexible chain with $\epsilon_{b}=$ $10^{2} k_{B} T$ respectively. Only the semi-flexible chain attains a noticeable helical conformation.

the experiments, here too the rigid chain (with lower $\mu$ value) shows a modest decrease in $R_{e e}$, in response to hydrophobic attraction. Chains of intermediate values of rigidity, i.e the chains with $\epsilon_{b}$ in the range conducive to helix formation, shows a comparatively larger decrease in the end to end distance as compared to the stiff chains. This is qualitatively (and quantitatively) similar to the experimental scenario presented in Fig.2 of the main text.

\section{D. $H 2$ for a polymeric chain with different degrees of hydrophobic compression.}

We show $H 2$ values as a function of $t / \tau$ for a semiflexible polymeric chain corresponding to different strengths of hydrophobic contractile forces on MGP partciles. To that end, we present data of $H 2$ versus $t / \tau$ where we vary $\Delta \ell_{c l}$ in units of $a$ which is the unit of length in our simulation. In the experiments, as the temperature is increased above the LCST temperature the magnitude of the hydrophobic forces increase in magnitude. In our model this effect can be incorporated by increasing $\Delta \ell_{c l}$. We note in Fig.S15, that $H 2$ (running averaged over $25 \tau$ ) shows an increase with increase in $\Delta \ell_{c l}$.

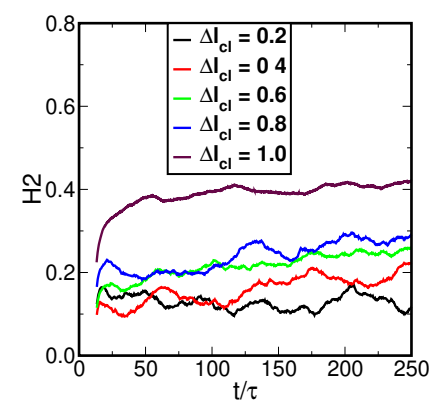

FIG. S15. H2 running averaged over $25 \tau$ as a function of time for $\kappa_{c l}=100 k_{B} T / a^{2}$ and $\epsilon_{b}=5000 k_{B} T(\mu \sim 0.8)$ under contractile forces due to hydrophobicity for various values of $\Delta \ell_{c l}$ as indicated by the legends. 


\section{REFERENCES}

(1) Banks, P. R.; Paquette, D. M. Comparison of Three Common Amine Reactive Fluorescent Probes Used for Conjugation to Biomolecules by Capillary Zone Electrophoresis. Bioconjugate chemistry 1995, 6, 447-458.

(2) Vutukuri, H. R.; Demirörs, A. F.; Peng, B.; van Oostrum, P. D. J.; Imhof, A.; van Blaaderen, A. Colloidal Analogues of Charged and Uncharged Polymer Chains with Tunable Stiffness. Angewandte Chemie International Edition 2012, 51, 11249-11253.

(3) Yethiraj, A. Tunable Colloids: Control of Colloidal Phase Transitions with Tunable Interactions. Soft Matter 2007, 3, 1099.

(4) Frisken, B. J. Revisiting the Method of Cumulants for the Analysis of Dynamic Light-Scattering Data. Applied Optics 2001, 40, 4087-4091.

(5) Mantelli, S.; Muller, P.; Harlepp, S.; Maaloum, M. Conformational Analysis and Estimation of the Persistence Length of DNA Using Atomic Force Microscopy in Solution. Soft Matter 2011, 7, 3412-3416. 